\title{
Propriocepção do joelho em indivíduos submetidos à menistectomia parcial e à sutura meniscal: estudo observacional
}

\author{
Knee proprioception in individuals submitted \\ to partial menistectomy and meniscal repair: \\ observational study
}

\section{Francklin Trindade da Silva' ${ }^{1}$ (1) Thiago Domingues Stocco ${ }^{2}$ (1)}

${ }^{1}$ Faculdade de Ciências Médicas da Santa Casa de São Paulo (São Paulo). São Paulo, Brasil. trindadefrank@gmail.com ²Autor para correspondência. Universidade Santo Amaro (São Paulo), Universidade Estadual de Campinas (Campinas). São Paulo, Brasil. tdstocco@live.com

\begin{abstract}
RESUMO | INTRODUÇãO: Os meniscos desempenham um papel fundamental na articulação, além de gerar estabilidade e congruência articular, influenciam na propriocepção do joelho. Lesões nesta estrutura muitas vezes só podem ser reparadas cirurgicamente, pelo fraco suprimento sanguíneo local, acarretando uma regeneração insatisfatória. A hipótese desse estudo é que possa existir menores déficits proprioceptivos em pacientes submetidos à sutura meniscal quando comparados aos submetidos à menistectomia parcial, devido a conservação da estrutura que tem importância proprioceptiva. OBJETIVO: Avaliar o déficit proprioceptivo do joelho em pacientes submetidos à menistectomia parcial e à sutura meniscal. METODOLOGIA: Estudo observacional transversal realizado na clínica de ortopedia e reumatologia da UNISA, em São Paulo. Participantes foram divididos em dois grupos. No Grupo I, indivíduos submetidos à menistectomia parcial, enquanto no Grupo II, a submetidos à sutura meniscal, sendo recrutados indivíduos de até 4 semanas de pós-operatório. Aprovado pelo comitê de ética seguido pelo CAAE 94144218.0.0000.0081. Para coleta dos dados foram realizados três testes: teste de senso de posicionamento ativo e passivo e teste de cinestesia. RESULTADOS: Participaram deste estudo uma amostra por conveniência de oito indivíduos (Grupo I, n=4 e Grupo 2, n=4), de ambos os gêneros, com média de idade de 33,4 anos ( \pm 11 anos). Os sujeitos do grupo I apresentaram maior déficit de proprioceptivo em comparação com o grupo II, nos testes realizados. Teste ativo do grupo I: $15^{\circ}=11,9^{\circ}$ $\pm 6,1 ; 30^{\circ}=11,6^{\circ} \pm 5,0 ; 45^{\circ}=9,4^{\circ} \pm 3,5$ e do grupo II, $15^{\circ}=7,6^{\circ} \pm 3,9$; $30^{\circ}=6,9^{\circ} \pm 2,8$. Teste de Cinestesia: Grupo I $132 \mathrm{~ms} \pm 51,5$ vs Grupo II 96ms $\pm 28,8$ ). CONCLUSÃo: Os meniscos demonstraram ser estruturas influentes para a propriocepção do joelho, apresentando maiores déficits em indivíduos que retiraram a estrutura.
\end{abstract}

PALAVRAS-CHAVE: Reabilitação. Propriocepção. Menisco.

\begin{abstract}
INTRODUCTION: Menisci play a fundamental role in the joint, in addition to generating joint stability and congruence, they influence knee proprioception. Lesions in this structure can often only be repaired surgically, due to the weak local blood supply, leading to unsatisfactory regeneration. The hypothesis of this study is that there may be less proprioceptive deficits in patients undergoing meniscal suture when compared to those undergoing partial menistectomy. OBJECTIVE: To evaluate the proprioceptive deficit of the knee in patients undergoing partial menistectomy and meniscal suture. METHODS: Cross-sectional observational study carried out at the UNISA orthopedics and rheumatology clinic in São Paulo. Participants were divided into two groups. In Group I, individuals who underwent partial menistectomy, while in Group II, who underwent meniscal suture, individuals were recruited up to 4 weeks after surgery. Approved by the ethics committee followed by CAAE 94144218.0 .0000 .0081 . For data collection, three tests were performed: test of active and passive sense positioning and kinesthesia test. RESULTS: Four individuals participated in Group I and four individuals in Group II, of both genders, between 20 and 40 years old. The results indicate that the patients who underwent partial menistectomy and meniscal suture showed proprioceptive differences between the operated and the non-operated limbs, however the subjects who had the preserved structure presented smaller values of difference in comparison with the operated members of the two groups (Active Test: $30^{\circ}=$ Group I $11.6^{\circ} \pm 5.0$ vs Group $116.9^{\circ} \pm$ 2.8, p = 0.010; $45^{\circ}=$ Group $19.4^{\circ} \pm 3.5$ vs Group II $6.5^{\circ} \pm 2.8, \mathrm{p}=$ $0.035 ; 60^{\circ}=$ Group $19.7^{\circ} \pm 4.3$ vs Group II $6.5^{\circ} \pm 4.9, p=0.103$; Kinesthesia Test: Group I $132 \mathrm{~ms} \pm 51.5$ vs Group II $96 \mathrm{~ms} \pm 28.8, \mathrm{p}$ $=0.046$ ). CONCLUSION: Higher proprioceptive deficits were found in subjects undergoing menistectomy compared to the sutured limb group, with a statistically significant difference.
\end{abstract}

KEYWORDS: Rehabilitation. Proprioception. Meniscus. 


\section{Introdução}

Os meniscos são descritos como discos fibrocartilaginosos de forma semilunar, localizados entre os côndilos da tíbia e do fêmur ${ }^{1}$. Cada joelho possui dois meniscos distintos: O menisco medial, em formato de " $\mathrm{C}$ ", menos móvel, cobrindo 51-71\% do platô tibial medial; e o menisco lateral, em formato de "O", que abrange $75-93 \%$ do platô tibial lateral².

Dentre as funções importantes do menisco destacam-se a transmissão e distribuição de carga, absorção de choque e aumento da congruência articular, além de ajudar a limitar o final dos movimentos de flexão e extensão, auxiliar na nutrição e lubrificação da articulação e contribuir na função proprioceptiva ${ }^{3}$.

A propriocepção pode ser definida como um conjunto de sensações aferentes, responsáveis pela sensação de movimento e posição articular, além de, influenciar respostas reflexas e controle motor voluntario e contribuir na estabilidade dinâmica da articulação, ajudando no papel de proteção, principalmente nos extremos da amplitude de movimento ${ }^{4,5}$.

Os sinais aferentes da propriocepção do joelho são enviados a partir de receptores mecânicos periféricos encontrados em estruturas articulares assim como nos músculos que envolvem a articulação. Estes mecanorreceptores atuam convertendo o estimulo mecânico de tensão ou compressão em impulsos elétricos específicos aferentes, enviando informações para o sistema nervoso central, podendo iniciar reflexos musculares de proteção ${ }^{6,7}$. Zimny et al. (1988), identificaram a presença de três tipos de mecanorreceptores no menisco medial humano: corpúsculos de Pacini, Terminações de Ruffini e Órgão Tendinoso de Golgi. Foi observado que os mecanorreceptores penetram parte do tecido perimeniscal seguindo para o terço externo e médio do corpo do menisco e para os cornos, onde foi demonstrada grande concentração, principalmente no corno posterior.

A presença de mecanorreceptores no menisco indica a importância dessa estrutura na função proprioceptiva e os déficits que uma lesão meniscal ou sua retirada cirúrgica, total ou parcial, poderiam causar para a estabilidade dinâmica da articulação, além dos efeitos deletérios como a aceleração do processo de degeneração da cartilagem articular levando ao início precoce da osteoartrite, principal doença articular, segunda maior causa de incapacidade física, gerando um profundo impacto socioeconômico ${ }^{9,10}$.

Nesse contexto, atualmente tem sido adotas técnicas para reparação tecidual, ao invés da menistectomia, (retirada parcial ou total do menisco) a sua conservação por técnica de sutura, mantendo o máximo de tecido intacto sempre que possível. No entanto, o procedimento de sutura do menisco em muitos casos é ineficaz ou impossível, e a retirada de tecido meniscal torna-se inevitável ${ }^{11}$. Por consequência, a menistectomia se apresenta como a intervenção cirúrgica mais comumente utilizada.

No Brasil, segundo dados do Ministério da Saúde de 2019, nos últimos cinco anos as menistectomias representaram aproximadamente $80 \%$ de todas as intervenções cirúrgicas para o tratamento de lesões meniscais.

Estudos anteriores observaram significante diminuição da função proprioceptiva do joelho em pacientes com lesões meniscais isoladas ${ }^{13}$ e submetidos à menistectomia parcial ${ }^{14}$. No entanto, nenhum estudo avaliou se existe uma diferença significante na propriocepção do joelho em pacientes submetidos à menistectomia parcial e à reparação meniscal por sutura.

Neste contexto, a hipótese desse estudo é que, baseado na literatura, possa existir uma diminuição significativa dos déficits causados na propriocepção do joelho de pacientes submetidos à sutura meniscal quando comparados aos submetidos à menistectomia parcial. Isso indicaria mais uma vantagem que reforça a ideia de preservar os meniscos do joelho através de sua reparação e, principalmente, a importância de se dar uma ênfase ainda maior na fase de treinamento proprioceptivo em pacientes submetidos à menistectomia, dentro de um programa de reabilitação.

Dessa forma, o objetivo desse estudo é avaliar comparativamente o déficit proprioceptivo do joelho em pacientes submetidos à menistectomia parcial e à sutura meniscal e portanto, observar a influência do menisco na propriocepção de joelho. 


\section{Materiais e métodos}

\section{Tipo de Pesquisa}

Esse trabalho refere-se a um estudo observacional transversal, descritivo, analítico e comparativo, de natureza quantitativa.

\section{Local de Pesquisa}

A pesquisa foi realizada no Ambulatório de Ortopedia e Reumatologia da clínica de fisioterapia da Universidade Santo Amaro (UNISA), localizada na Rua Professor Enéas de Siqueira Neto, 340 - Jardim das Imbuias, São Paulo - SP, no ano de Set/ 2018Set/2019, período no qual os pacientes foram recrutados e avaliados seguindo protocolo de avaliação descrito adiante.

A pesquisa foi iniciada após a aprovação pelo Comitê de Ética e Pesquisa da universidade, sob o parecer 2.887.319 e CAAE 94144218.0.0000.0081.

\section{Casuística}

\section{Amostra}

Este estudo incluiu uma amostra por conveniência de oito indivíduos de ambos os sexos ( 3 mulheres e 5 homens), com média de idade de 33,4 anos ( $\pm 11,4$ anos), previamente submetidos à procedimentos cirúrgicos para tratamento de lesões isoladas no menisco, sendo divididos em dois grupos: Grupo I e Grupo II. No Grupo I, foram incluídos quatro indivíduos submetidos à menistectomia parcial do menisco, enquanto no Grupo II, quatro sujeitos onde a técnica de escolha para o tratamento da lesão foi a sutura meniscal.

\section{Critérios de Inclusão}

Como critério de inclusão os indivíduos que concordassem com o Termo de Consentimento Livre Esclarecido foram selecionados com a faixa etária entre 20 e 40 anos de idade ${ }^{15}$, ter até quatro semanas de pós- operatório, precisando apresentar no mínimo $90^{\circ}$ de flexão do joelho e já não apresentar sinais de inflamação, como dor e edema.

\section{Critérios de Exclusão}

Foram excluídos da pesquisa indivíduos que apresentaram déficits cognitivos, que fossem portadores de outras lesões ou disfunções no joelho ou em outro segmento do corpo que prejudicasse a avaliação ou que realizaram previamente algum tipo de treinamento proprioceptivo após a cirurgia.

\section{Procedimento Experimental}

\section{Avaliação da propriocepção do joelho}

Para mensurar a propriocepção do joelho foram realizados o método de avaliação do senso de posicionamento e avaliação da cinestesiaa ${ }^{16-18}$.

a) Avaliação do senso de posicionamento

O teste de avaliação do senso de posicionamento para mensurar a propriocepção do joelho é dividido em dois métodos: Teste ativo e teste passivo.

\section{- $\quad$ Teste ativo}

A mensuração da propriocepção pelo método ativo da avaliação do senso de posicionamento se baseia na capacidade do indivíduo de mover ativamente a articulação do joelho para um angulo pré-definido sem o auxílio do sistema visual. Para tanto, a articulação do joelho do indivíduo vendado é previamente movida pelo examinador para um ângulo alvo e retornada passivamente para posição inicial. Posteriormente, o examinador solicitou que o indivíduo movesse seu membro inferior ativamente de forma a reproduzir o ângulo anteriormente demonstrado.

Antes de realizar os testes definitivos o indivíduo foi instruído a fazê-los sem venda nos olhos, para não haver erro de entendimento que prejudicasse os resultados.

Após a explicação, o paciente foi vendado e o examinador posicionou sua perna em angulações da articulação do joelho pré-determinadas $\left(15^{\circ}, 30^{\circ}, 45^{\circ}\right.$ e $\left.60^{\circ}\right)$ por 10 segundos em posição estática. Em seguida, foi solicitado ao indivíduo que realizasse o mesmo movimento de forma ativa parando na mesma angulação pré-estabelecida, conforme descrição anterior do teste. 
Os testes foram repetidos três vezes em cada ângulo e de forma não sequencial, tanto no membro submetido à cirurgia quanto o membro contralateral

Dessa forma, foi avaliada a capacidade do indivíduo de definir o ângulo da posição articular do joelho, mensurando a diferença entre o ângulo real da articulação com a sensação do ângulo percebido pelo paciente, além da comparação contralateral.

\section{- Teste passivo}

Semelhante ao teste ativo, na avaliação do senso de posicionamento pelo método passivo, a articulação do joelho do indivíduo vendado também foi previamente movida pelo examinador para um ângulo alvo pré-determinado e retornada passivamente para posição inicial. No entanto, em seguida, o examinador moveu passivamente a articulação em uma velocidade de aproximadamente $5 \%$ e o indivíduo então foi solicitado a indicar quando tivesse a sensação que a articulação do joelho atingiu o ângulo alvo demonstrado anteriormente. A diferença entre o ângulo indicado pelo indivíduo e o ângulo alvo foi registrada.

Da mesma forma que o teste ativo, os testes foram repetidas três vezes em cada ângulo da articulação do joelho $\left(15^{\circ}, 30^{\circ}, 45^{\circ}\right.$ e $\left.60^{\circ}\right)$ de forma não sequencial nos dois membros inferiores.

\section{b) Avaliação da Cinestesia}

Ao contrário da avaliação de propriocepção pela posição da articulação no espaço, a cinestesia e denominada por detectar a percepção de movimento articular. A avaliação da cinestesia foi mensurada a partir do movimento articular passivo, onde o avaliador segurou a porção distal do membro inferior do paciente por um tempo não determinado, e então, o movimentou. $\mathrm{O}$ paciente esteve sentado e vendado, $\mathrm{e}$ assim que percebeu o movimento articular do joelho ele indicou. $O$ tempo até a percepção do movimento foi registrado por um cronômetro digital.

\section{Procedimentos para aquisição dos dados}

Para a mensuração da angulação da articulação do joelho foi utilizado a Fotogrametria computadorizada, técnica de análise de imagens fotográficas para obtenção da posição articular ${ }^{19}$. Para tanto, as imagens foram capturadas por uma câmera digital (Sony
Cyber- Shot DSC-W35, 7.2 megapixels). Os indivíduos ficaram posicionados em decúbito ventral na maca sendo demarcados com três marcadores auto adesivos localizados sobre o maléolo lateral, côndilo lateral do fêmur e trocânter maior do fêmur, a fim de estipular pontos de referências para as análises posteriores. A câmera ficou posicionada em um suporte próprio com a altura sendo normalizada alinhando-a com o joelho do indivíduo e afastado a uma distância de 1,5 metros.

Em seguida, para mensuração da amplitude de mo vimento da articulação do joelho, as imagens capturadas foram processadas e analisadas no computador utilizando o software ImageJ (Figura 1) $(\mathrm{NIH}$, http://rsb.info.nih.gov/ij).

Figura 1. Análise de angulação do joelho através da fotogrametria computadorizada

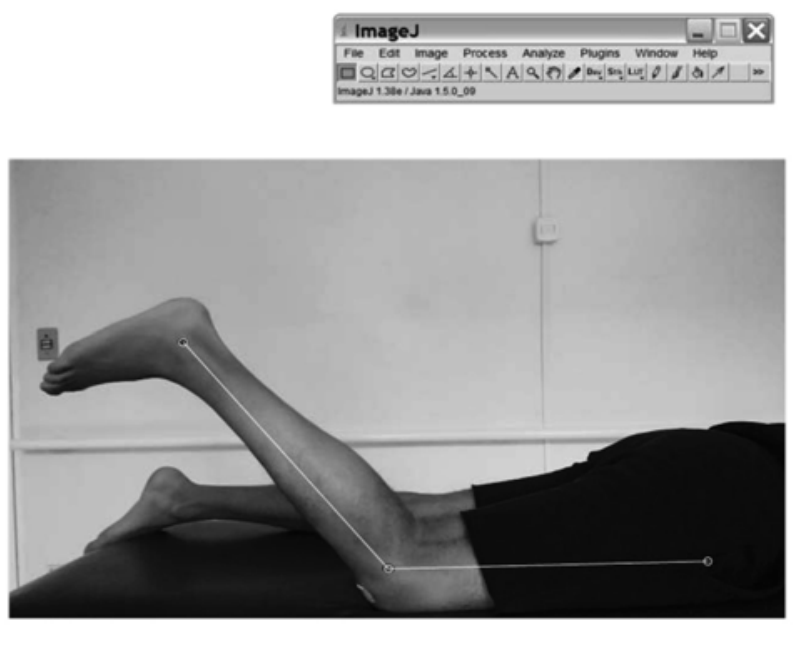

Fonte: Os autores (2020).

\section{Análises Estatísticas}

A partir dos valores obtidos utilizou-se a estatística descritiva para apresentar os resultados e comparar os dados amostrais. Os resultados foram expressos como média e desvio padrão e os testes estatísticos adequados aplicados: $O$ teste $t$ Student não pareado foi realizado para comparar os dados das médias da angulação alcançada em comparação com o membro contralateral e entre os membros operados dois dois grupos. Para o teste, o nível de significância foi de $95 \%(p<0,05)$. Foi utilizado o software estatístico Minitab ${ }^{\circ}$ (versão 19, Minitab Inc., StateCollege, EUA). 


\section{Resultados}

Os resultados obtidos no teste de senso de posicionamento ativo e passivo mostram que, os indivíduos submetidos à menistectomia parcial (Grupo I) apresentam diferenças entre o ângulo alvo e o ângulo aferido em grande parte dos ângulos analisados, demonstrando alteração proprioceptiva em comparação com o membro não operado, com diferença estatisticamente significante na maioria dos ângulos analisados (Tabela 1).

Tabela 1. Teste de senso de posicionamento ativo e passivo de indivíduos submetidos à menisctectomia (Grupo I)- Valor de diferença do ângulo aferido em relação ao ângulo alvo, entre os membros operado e o não operado

\begin{tabular}{|c|c|c|c|c|c|}
\hline \multicolumn{2}{|c|}{ Grupo I } & \multicolumn{4}{|c|}{ Ângulo Alvo } \\
\hline & & $15^{\circ}$ & $30^{\circ}$ & $45^{\circ}$ & $60^{\circ}$ \\
\hline \multirow[t]{3}{*}{ Teste ativo } & Op & $11,9^{\circ}( \pm 6,1)$ & $11,6^{\circ}( \pm 5,0)$ & $9,4^{\circ}( \pm 3,5)$ & $9,7^{\circ}( \pm 4,3)$ \\
\hline & Nop & $4,0^{\circ}( \pm 2,4)$ & $2,8^{\circ}( \pm 2,5)$ & $7,0^{\circ}( \pm 4,8)$ & $3,2^{\circ}( \pm 2,5)$ \\
\hline & & $p=<0,001$ & $p=<0,001$ & $p=0,130$ & $p=<0,001$ \\
\hline \multirow[t]{3}{*}{ Teste passivo } & Op & $6,5^{\circ}( \pm 4,6)$ & $6,6^{\circ}( \pm 3,1)$ & $8,0^{\circ}( \pm 3,2)$ & $10,3^{\circ}( \pm 5,4)$ \\
\hline & Nop & $2,3( \pm 1,9)$ & $1,8^{\circ}( \pm 1,2)$ & $2,3^{\circ}( \pm 1,8)$ & $3,5^{\circ}( \pm 2,2)$ \\
\hline & & $p=0,007$ & $p=<0,001$ & $p=<0,001$ & $p=<0,001$ \\
\hline
\end{tabular}

Op= Operado; Nop= Não operado;

Autor

Os valores obtidos no teste de senso de posicionamento passivo, em indivíduos submetidos à sutura meniscal (Grupo II), apresentam diferenças entre o ângulo alvo e o ângulo aferido, demostrando alteração proprioceptiva em comparação com o membro não operado. Entretanto, a partir ângulo de $60^{\circ}$, no teste de senso de posicionamento passivo, não houve diferença significante da propriocepção do joelho comparado com o membro não operado (Tabela 2).

Tabela 2. Teste de senso de posicionamento ativo e passivo de indivíduos submetidos à sutura de menisco (Grupo II)- Valor de diferença do ângulo aferido em relação ao ângulo alvo, entre os membros operado e o não operado

\begin{tabular}{cccccc}
\hline \multicolumn{2}{c}{ Grupo I } & \multicolumn{5}{c}{ Ângulo Alvo } \\
\hline Teste ativo & \multicolumn{1}{c}{$\mathbf{1 5}^{\circ}$} & $\mathbf{3 0}^{\circ}$ & $\mathbf{4 5}^{\circ}$ & $\mathbf{6 0}^{\circ}$ \\
& Op & $11,9^{\circ}( \pm 6,1)$ & $11,6^{\circ}( \pm 5,0)$ & $9,4^{\circ}( \pm 3,5)$ & $9,7^{\circ}( \pm 4,3)$ \\
& Nop & $4,0^{\circ}( \pm 2,4)$ & $2,8^{\circ}( \pm 2,5)$ & $7,0^{\circ}( \pm 4,8)$ & $3,2^{\circ}( \pm 2,5)$ \\
Teste passivo & & $p=<0,001$ & $p=<0,001$ & $p=0,130$ & $p=<0,001$ \\
& Op & $6,5^{\circ}( \pm 4,6)$ & $6,6^{\circ}( \pm 3,1)$ & $8,0^{\circ}( \pm 3,2)$ & $10,3^{\circ}( \pm 5,4)$ \\
& Nop & $2,3( \pm 1,9)$ & $1,8^{\circ}( \pm 1,2)$ & $2,3^{\circ}( \pm 1,8)$ & $3,5^{\circ}( \pm 2,2)$ \\
& & $p=0,007$ & $p=<0,001$ & $p=<0,001$ & $p=<0,001$ \\
\hline
\end{tabular}

Op= Operado; Nop= Não operado;

Autor

Os resultados apresentados no teste de cinestesia mostram que, em indivíduos submetidos à sutura de menisco (Grupo II), há percepção reduzida do movimento, comparado aos indivíduos submetidos à menistectomia (Grupo I), com resultados estatisticamente significantes (Tabela 3).

Tabela 3. Teste de cinestesia - comparação entre o tempo médio de resposta ao movimento do membro operado e não operado de indivíduos submetidos à menistectomia parcial (Grupo I) e sutura de menisco (Grupo II)

\begin{tabular}{cccc}
\hline & \multicolumn{2}{c}{ Tempo Médio (ms) } \\
\hline & Membro operado & Membro não operado & Valor $\boldsymbol{p}$ \\
\hline Grupo I & $132( \pm 51,5)$ & $87( \pm 32,7)$ & 0,186 \\
Grupo II & $96( \pm 28,8)$ & $60( \pm 10,0)$ & $<0,001$ \\
\hline ms= milissegundos; & & Autor
\end{tabular}


Os resultados obtidos no teste de senso de posicionamento ativo mostram que, os indivíduos pós sutura (Grupo II) apresentaram menores déficits proprioceptivos em relação aos submetidos à menistectomia (Grupo I), em comparação com os membros operados, em três dos quatro ângulos avaliados (Gráfico 1).

Gráfico 1. Teste de senso de posicionamento ativo de indivíduos pós menistectomia parcial (Grupo I) e sutura de menisco (Grupo II)- Média da diferença entre o ângulo de referência e o ângulo aferido dos membros operados; $15^{\circ}=$ Grupo I vs Grupo II $(p=0,024) ; 30^{\circ}=$ Grupo I vs Grupo II ( $\left.p=0,010\right) ; 45^{\circ}=$ Grupo I vs Grupo II $(p=0,035) ; 60^{\circ}=$ Grupo I vs Grupo II $(p=0,103)$

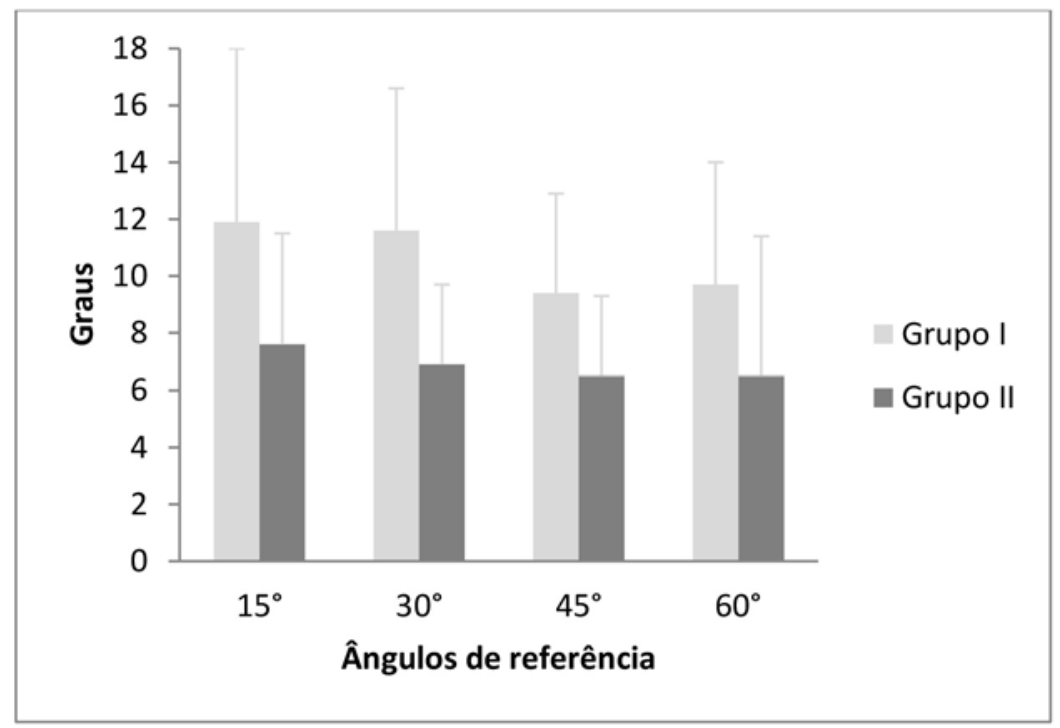

Embora não haja diferença estatisticamente significante, os resultados obtidos no teste de senso de posicionamento passivo mostram que, os indivíduos submetidos à menistectomia apresentaram maior diferença no membro operado em três dos quatro ângulos analisados, em comparação com os indivíduos suturados (Gráfico 2).

Gráfico 2. Teste de senso de posicionamento passivo de indivíduos pós menistectomia parcial (Grupo I) e sutura de menisco (Grupo II)- Média da diferença entre o ângulo de referência e o ângulo aferido dos membros operados; $15^{\circ}=$ Grupo I vs Grupo II $(p=0,380) ; 30^{\circ}=6 r u p o ~ I$ vs $G$ rupo II $(p=0,943) ; 45^{\circ}=$ Grupo I vs Grupo II $(p=0,764) ; 60^{\circ}=$ Grupo I vs Grupo II $(p=0,128)$

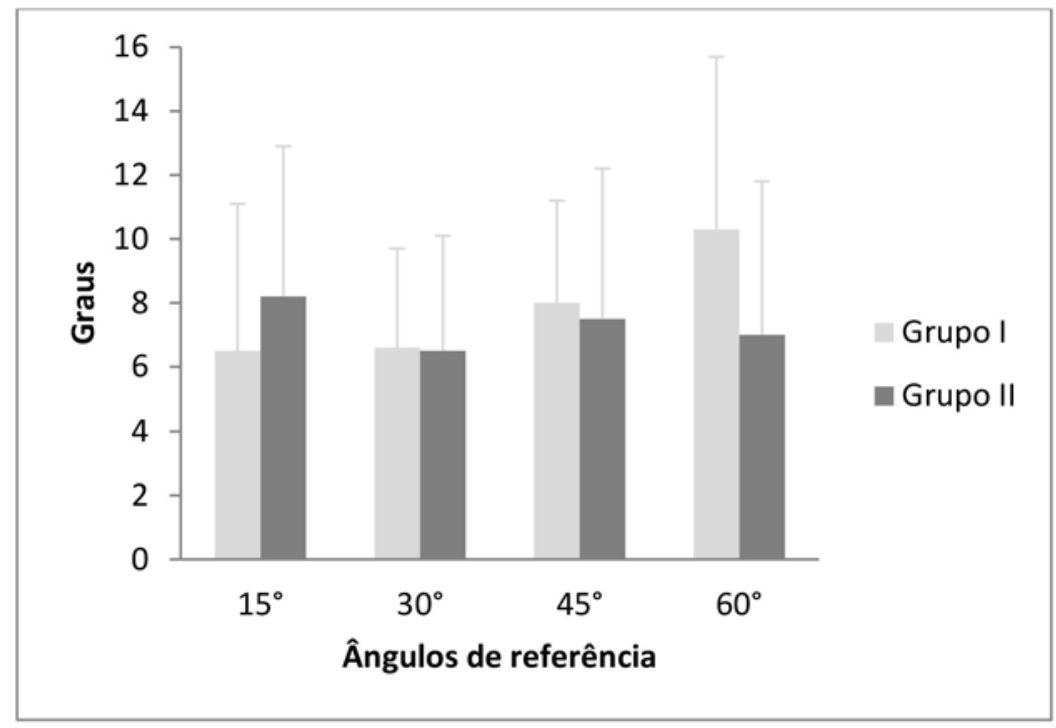

Os resultados obtidos no teste de senso de posicionamento ativo mostram que, os indivíduos submetidos à menistectomia parcial (Grupo I) apresentaram maior tempo de resposta ao movimento em comparação com os submetidos à sutura de menisco (Grupo II), com diferença estatisticamente significante (Tabela 4). 
Tabela 4. Teste de cinestesia - comparação da diferença do tempo de resposta ao movimento entre membros operados de indivíduos submetidos à menistectomia parcial (Grupo I) e a sutura de menisco (Grupo II)

\begin{tabular}{cccc}
\hline \multicolumn{4}{c|}{ Tempo Médio $(\mathbf{m s})$} \\
\hline Grupo I & Grupo II & Valor $\boldsymbol{p}$ \\
\hline Membro operado & $132( \pm 51,5)$ & $96( \pm 28,8)$ & 0,046 \\
\hline ms= milissegundos; & & Autor &
\end{tabular}

\section{Discussão}

O presente estudo objetivou analisar quantitativamente e comparativamente a propriocepção do joelho em indivíduos submetidos à menistectomia parcial e a sutura de menisco, através de três testes.

Os resultados evidenciam que há redução da propriocepção nos indivíduos pós menistectomia parcial e sutura de menisco, sendo maior em indivíduos submetidos à menistectomia parcial, em dois dos três testes realizados.

Foram encontradas diferenças da propriocepção do joelho pós menistectomia parcial e a sutura meniscal, em comparação com o membro não operado apresentando resultados estatisticamente significantes em $94 \%$ dos ângulos analisados nos testes de senso de posicionamento ativo e passivo.

Observou-se que os resultados encontrados em indivíduos submetidos à menistectomia parcial divergem com um estudo anterior ${ }^{20}$ que avaliou a propriocepção do joelho através do teste de senso de posicionamento passivo, em pacientes com pós operatório de seis meses após menistectomia parcial. Os autores observaram que não houve diferenças na propriocepção e comparação com o grupo controle. Contudo, os participantes tiveram um maior tempo até a análise, fazendo com que haja maior cicatrização do tecido. Um dado importante a ser analisado é que o trabalho não evidencia se os participantes realizaram algum tipo de reabilitação.

Evidenciou-se diferenças proprioceptivas, nos três testes analisados, em participantes submetidos à sutura de menisco, em comparação com o membro não operado. Contudo, a análise do ângulo de 60 graus no teste de senso de posicionamento passivo, mostrou-se não haver diferença estatisticamente significante entre o membro não operado, ou seja, não houve déficit da propriocepção. Isso pode estar relacionado com uma maior pressão sobre o menisco nesse ângulo que meIhora a resposta aferente dos mecanorreceptores presentes na estrutura, corroborando com o estudo feito por Karahan ${ }^{21}$, no qual foram encontrados maiores déficits proprioceptivos em ângulos maiores ou iguais a 60 graus, mostrando uma importância da integridade da estrutura para melhor resposta aferente durante atividades funcionais ${ }^{22}$.

O processo de reabilitação nos dois procedimentos cirúrgicos avaliados é diferente, principalmente no que se diz respeito à descarga de peso, onde em um procedimento como a menistectomia parcial é realizada geralmente na primeira semana conforme tolerado pelo indivíduo e, em pacientes submetidos à sutura de menisco têm um tempo maior até a descarga de peso total com a média em torno de 4-6 semanas, pois deve-se respeitar o processo de regeneração da estrutura suturada ${ }^{23}$. Sabe-se também que, um tempo prolongado de imobilização leva a prejuízos neuromusculares e de resposta proprioceptiva, devido uma relação à resposta aos estímulos de tração ou coaptação articular encontradas nos mecanorreceptores, esse mecanismo de resposta ao estímulo pode prejudicar os sujeitos submetidos à abordagem cirúrgicas que ficam sem a descarga de peso. Portanto, ao analisarmos sujeitos submetidos à sutura meniscal nesse presente estudo, durante o teste de cinestesia, os resultados mostram que, em relação ao membro não operado, esses efeitos deletérios estão presentes nos sujeitos submetidos à sutura de menisco. Em média, esses indivíduos apresentaram descarga de peso parcial com utilização de muletas e imobilizador de joelho nas primeiras duas semanas, corroborando com os resultados apresentados. Isso também pode ser explicado porque não há diferença dos membros operados nos sujeitos pós menistectomia parcial e sutura de menisco, em relação ao teste de senso de posicionamento passivo, onde há uma maior demanda proprioceptiva da estrutura ${ }^{24}$. 
Entretanto, a análise dos resultados apresentados pelos sujeitos do submetidos à menistectomia parcial em comparação com os suturados demonstram que, mesmo os indivíduos pós menistectomia parcial apresentarem descarga de peso precoce, mostraram- se com piores resultados proprioceptivos com diferenças estatisticamente significantes da propriocepção entre os membros operados, ou seja, há uma influência proprioceptiva do menisco e o que sua retirada pode afetar na articulação em relação ao tempo de resposta ${ }^{25-28}$.

Sendo os meniscos do joelho estruturas importantes para a função proprioceptiva, a retirada após a abordagem por menistectomia parcial influenciaria diretamente na propriocepção e na cinemática da articulação, corroborando com os resultados apresentados no presente estudo 29 .

Com auxílio dos dados apresentados podemos compreender a importância do treinamento proprioceptivo em pacientes submetidos à esses tipos cirúrgicos e de se iniciar a reabilitação neuromuscular funcional de forma precoce, como na implementação de exercícios de equilíbrio, força e resistência muscular, mobilização articular ativa ou manual. Alguns de exercícios que podem ser aplicados incluem os trabalhos com treino em apoio unipodal e até a utilização de bases instáveis, onde o corpo necessitaria do sistema proprioceptivo para realizar tal tarefa ${ }^{26,27}$.

Uma das dificuldades encontrada no presente estudo foi achar pacientes que condigam com critérios de inclusão, principalmente, ao se tratar de lesões e/ ou procedimentos cirúrgicos como suturas isoladas de menisco que muitas vezes, devido ao seu mecanismo de lesão (flexão de joelho e força rotacional), pode levar à ruptura parcial ou total de outras estruturas, comumente associado ao ligamento cruzado anterior 30. Portanto, a amostra reduzida nesse estudo representa um potencial viés dos resultados.

O presente trabalho torna-se o primeiro estudo a avaliar comparativamente a propriocepção de joelho em indivíduos submetidos à menistectomia parcial e a sutura meniscal, com resultados potenciais que podem ser explorados em estudos futuros, com um número maior de participantes e, até mesmo uma comparação em indivíduos com maior tempo de cirurgia, tipo cirúrgico específico correlacionando a quantidade de tecido retirado em relação a perda de propriocepção e, após a alta da reabilitação.

\section{Conclusão}

Os meniscos do joelho demonstraram ser estruturas muito influentes para a propriocepção do joelho nos resultados apresentados.

Foram encontradas diferenças da propriocepção do joelho pós menistectomia parcial, em comparação com o membro não operado em dois dos três testes realizados, diferente dos indivíduos submetidos à sutura de menisco, que foram nos três testes realizados.

Ao comparar os resultados de propriocepção em relação ao membro operado dos dois grupos, os indivíduos submetidos à menistectomia parcial mostraram-se com maiores déficits proprioceptivos em relação ao grupo de sutura. Entretanto, como os resultados demonstraram diferenças de propriocepção nos dois grupos, deve-se dar ênfase aos estímulos neuromusculares o mais precocemente para amenizar efeitos deletérios em longo prazo, tanto nos pacientes submetidos à menistectomia quanto nos submetidos à sutura de menisco.

\section{Contribuições dos autores}

Silva FT participou da coleta dos dados da pesquisa, análise estatística dos dados da pesquisa, interpretação dos resultados e redação. Stocco TD participou da concepção, delineamento, análise estatística dos dados da pesquisa, interpretação dos resultados e redação do artigo científico

\section{Conflitos de interesses}

Nenhum conflito financeiro, legal ou político envolvendo terceiros (governo, empresas e fundações privadas, etc.) foi declarado para nenhum aspecto do trabalho submetido (incluindo, mas não se limitando a subvenções e financiamentos, participação em conselho consultivo, desenho de estudo, preparação de manuscrito, análise estatística, etc.).

\section{Referências}

1. Renstrom $\mathrm{P}$, Johnson RJ. Anatomy and biomechanics of the menisci. Clin Sport Med. 1990;9(3):523-538.

2. Clark CR, Ogden JA. Development of the menisci of the human knee joint. Morphological changes and their potential role in childhood meniscal injury. J Bone Joint Surg Am. 1983;65(4):538547. doi: $10.2106 / 00004623-198365040-00018$ 
3. Barber FA. Accelerated rehabilitation for meniscus repairs. Arthroscopy. 1994;10(2):206-210. doi: 10.1016/507498063(05)80095-7

4. Gray JC. Neural and vascular anatomy of the menisci of the human knee. J Orthop Sports Phys Ther. 1999;29(1):23-30. doi: $\underline{10.2519 / j o s p t .1999 .29 .1 .23}$

5. Saygi B, Yildirim Y, Berker N, Ofluoglu D, Karadag-Saygi E, Karahan M. Evaluation of the neurosensory function of the medial meniscus in humans. Arthroscopy. 2005;21(12):1468-1472. doi: 10.1016/j.arthro.2005.09.006

6. Solomonow M, Baratta R, Zhou BH, Shoji H, Bose W, Beck C et al. The synergistic action of the anterior cruciate ligament and thigh muscles in maintaining joint stability. Am J Sports Med. 1987;15(3):207-213. doi: 10.1177/036354658701500302

7. Chahla J, Cinque ME, Godin JA, G Sanchez, Lebus GF, Whalen $J M$ et al. Meniscectomy and Resultant Articular Cartilage Lesions of the Knee Among Prospective National Football League Players: An Imaging and Performance Analysis. Am J Sports Med. 2018;46(1):200-207. doi: 10.1177/0363546517737991

8. Zimny ML, Albright DJ, Dabezies E. Mechanoreceptors in the human medial meniscus. Acta Anat (Basel). 1988;133(1):35-40. doi: 10.1159/000146611

9. Fox AJS, Wanivenhaus F, Burge AJ, Warren RF, Rodeo SA The human meniscus: A review of anatomy, function, injury, and advances in treatment. Clin Anat. 2015;28(2):269-287. doi: 10.1002/ca.22456

10. Hunter DJ, Schofield D, Callander E. The individual and socioeconomic impact of osteoarthritis. Nat Rev Rheumatol. 2014;10(7):437-41. doi: 10.1038/nrrheum.2014.44

11. Abrams GD, Frank RM, Gupta AK, Harris JD, McCormick FM, Cole BJ. Trends in meniscus repair and meniscectomy in the United States, 2005-2011. Am J Sports Med. 2013;41(10):23332339. doi: $10.1177 / 0363546513495641$

12. DATASUS - Departamento de Informática do SUS. Procedimentos Hospitalares do SUS - por local de internação Brasil. [Internet]. [acesso em 2020 abr. 24]. Disponível em: http:// tabnet.datasus.gov.br/cgi/tabcgi.exe?sih/cnv/qiuf.def

13. Jerosch J, Prymka M. [Proprioceptive deficits of the knee joint after rupture of the medial meniscus]. Unfallchirurg. 1997;100(6):444-448. doi: 10.1007/s001130050140

14. Malliou P, Gioftsidou A, Pafis G, Rokka S, Kofotolis N, Mavromoustakos $S$ et al. Proprioception and functional deficits of partial meniscectomized knees. Eur J Phys Rehabil Med. 2012;48(2):231-236.

15. Cossich V, Mallrich F, Titonelli V, Sousa EB, Velasques B, Salles Jl. Déficit proprioceptivo em indivíduos com ruptura unilateral do ligamento cruzado anterior após a avaliação ativa do senso de posição articular. Rev Bras Ortop. 2014;49(6):607-612. doi: 10.1016/j.rbo.2013.07.009
16. Alonso AC, Brech GC, Greve JMD. Técnicas de avaliação proprioceptiva do ligamento cruzado anterior do joelho. Acta fisiatr. 2010;17(3):134-140.

17. Mir SM, Hadian M-R, Talebian S, Nasseri N. Functional assessment of knee joint position sense following anterior cruciate ligament reconstruction. Br J Sports Med. 2008;42(4):300303. doi: $10.1136 /$ bjsm.2007.044875

18. Kim H-J, Lee J-H, Lee D-H. Proprioception in Patients With Anterior Cruciate Ligament Tears. Am J Sports Med. 2017;45(12):2916-2922. doi: 10.1177/0363546516682231

19. Barbosa GM, Santos HH, Dantas GAF, Silva BR, Pinheiro SM, Vieira WHB. Intra-rater and inter-instrument reliability on range of movement of active Knee extension. Motriz Rev Educ Fis. 2017;23(1):53-59. doi: 10.1590/S1980-6574201700010008

20. Jerosch J, Prymka M, Castro WH. Proprioception of knee joints with a lesion of the medial meniscus. Acta Orthop Belg. 1996;62(1):41-45.

21. Karahan M, Kocaoglu B, Cabukoglu C, Akgun U, Nuran R. Effect of partial medial meniscectomy on the proprioceptive function of the knee. Arch Orthop Trauma Surg. 2010;130(3):427-431. doi: 10.1007/s00402-009-1018-2

22. Fremerey RW, Lobenhoffer P, Zeichen J, Skutek M, Bosch U, Tscherne $\mathrm{H}$. Proprioception after rehabilitation and reconstruction in knees with deficiency of the anterior cruciate ligament. J Bone Jt Surg. 2000;82(6):801-806. doi: 10.1302/0301-620X.82B6.10306

23. Cavanaugh JT, Killian SE. Rehabilitation following meniscal repair. Current Rev Musculoskelet Med. 2012;5(1):46-58. doi: 10.1007/s12178-011-9110-y

24. Chen S, Fu P, Wu H, Pei M. Meniscus, articular cartilage and nucleus pulposus: a comparative review of cartilage-like tissues in anatomy, development and function. Cell Tissue Res. 2017;370(1):53-70. doi: 10.1007/s00441-017-2613-0

25. Caplan N, Forbes A, Radha S, Stewart S, Ewen A, Gibson ASC et al. Effects of 1 week of unilateral ankle immobilization on plantar-flexor strength, balance, and walking speed: A pilot study in asymptomatic volunteers. J Sport Rehabil. 2015;24(2):156-162. doi: $10.1123 / \mathrm{jsr} .2013-0137$

26. Zhang X, Hu M, Lou Z, Liao B . Effects of strength and neuromuscular training on functional performance in athletes after partial medial meniscectomy. J Exerc Rehabil. 2017;13(1):110116. doi: $10.12965 /$ jer.1732864.432

27. Clark NC, Roijezon U, Treleaven J. Proprioception in musculoskeletal rehabilitation. Part 2: Clinical assessment and intervention. Man Ther. 2015;20(3):378-387. doi: 10.1016/j. math.2015.01.009 
28. Kunz RI, Silva LI, Costa JRG, Soares CLR, Bertolini GRF, Brancalhão RMC, et al. Alterações histomofometricas na articulação do joelho de ratos Wistar após remobilização em meio aquático. Fisioter Pesq. 2015;22(3):317-324 doi: 10.590/18092950/14234922032015

29. LaPrade CM, James EW, Cram TR, Feagin JA, Engebretsen L, Laprade RF. Meniscal Root Tears: A Classification System Based on Tear Morphology. Am J Sports Med. 2015;43(2):363-369. doi: $\underline{10.1177 / 0363546514559684}$

30. Gaillard R, Magnussen R, Batailler C, Neyret P, Lustig S, Servien E. Anatomic risk factor for meniscal lesion in association with $\mathrm{ACL}$ rupture. J Orthop Surg Res. 2019;14(1):242. doi: 10.1186/s13018019-1281-z 